\title{
A note on weak convergence results for infinite causal triangulations
}

\author{
Valentin Sisko $^{\mathrm{a}}$, Anatoly Yambartsev ${ }^{\mathrm{b}}$ and Stefan Zohren ${ }^{\mathrm{c}, 1}$ \\ ${ }^{a}$ Universidade Federal Fluminense \\ ${ }^{\mathrm{b}}$ University of São Paulo \\ ${ }^{\mathrm{c}}$ Oxford University
}

\begin{abstract}
We discuss infinite causal triangulations and equivalence to the size biased branching process measure- the critical Galton-Watson branching process distribution conditioned on non-extinction. Using known results from the theory of branching processes, this relation is used to prove a novel weak convergence result of the joint length-area process of a infinite causal triangulations to a limiting diffusion. The diffusion equation enables us to determine the physical Hamiltonian and Green's function from the FeynmanKac procedure, providing us with a mathematical rigorous proof of certain scaling limits of causal dynamical triangulations.
\end{abstract}

\section{Introduction}

Models of planar random geometry provide a rich field with an interplay between mathematical physics and probability.

On the physics side so-called dynamical triangulations (DT) have been introduced as models for two-dimensional Euclidean quantum gravity and string theory (see, e.g., Ambjørn, Durhuus and Jonsson (1997) for an overview). The basic idea is to define the gravitational path integral as a sum over triangulated surfaces. Any physical observable is then defined on the ensemble of all such triangulations. At the end, continuum physics is obtained by performing a scaling limit in which one takes the size of the triangulations to infinity keeping the physical area constant.

On the probabilistic side Angel and Schramm (2003) first introduced the uniform measure on infinite planar triangulations proving the existence of the above scaling limit as a weak limit. This construction was essential to prove several properties of such uniform infinite triangulations. In particular Angel (2003) proved that the volume of a ball $B(R)$ of radius $R$ is of order $R^{4}$ and that the length of the boundary is of order $R^{2}$. This proved rigorously that the fractal dimension of such

\footnotetext{
${ }^{1}$ This work was done while the author was at the Mathematical Institute, Leiden University, The Netherlands and the Department of Statistics, University of São Paulo, Brazil.

Key words and phrases. Causal triangulation, scaling limits, weak convergence, diffusion process, branching process.

Received November 2015; accepted February 2017.
} 


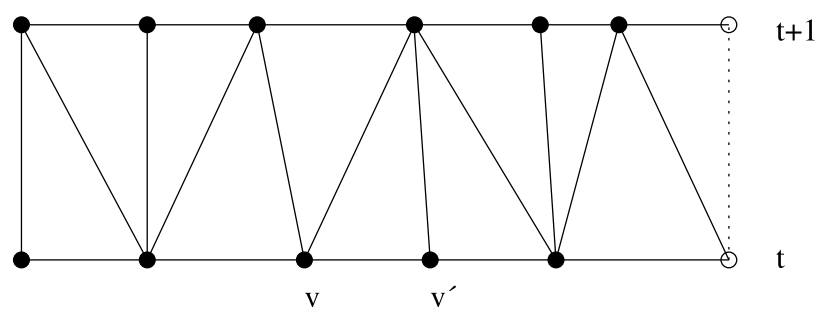

Figure 1 A time slice from time t to time $t+1$. The left and right side of the strip should be identified to form a band with topology $S^{1} \times[t, t+1]$.

triangulations is $d_{H}=4,{ }^{2}$ a result long known to physicists (see, e.g., Ambjørn, Durhuus and Jonsson (1997)). Later Krikun (2005) obtained the exact limit theorem for the scaled boundary length of $B(R)$.

While two-dimensional Euclidean quantum gravity defined through DT definitely has a rich mathematical structure as pointed out above, as a model of quantum gravity it failed to be numerically extended to higher dimensions. This leads to the development of a different approach of so-called Causal Dynamical Triangulations (CDT) by Ambjørn and Loll (1998). In contrast to the Euclidean model, CDT provides a nonperturbative definition of the Lorentzian gravitational path integral (the informal definition of gravitational path integral for mathematicians see, for example, the introduction in Napolitano and Turova (2016)). These causal triangulations differ from their Euclidean analogs in the fact that they have a timesliced structure of fixed spatial topology. Here we consider triangulations of an overall topology of a cylinder (extensions to other topologies are straightforward). Then the triangulation consists of slices $S^{1} \times[t, t+1]$ from time $t$ to time $t+1$ as illustrated in Figure 1. Here, edges connecting vertices in slices of equal time are called space-like edges, while edges connecting subsequent slices are called time-like edges.

Note that this class of triangulations forms a causal structure needed to model Lorentzian geometries. In particular, we can think that a vertex $v^{\prime}$ lies in the future of a vertex $v$, that is, vertices $v$ and $v^{\prime}$ are causally related, iff there is a path of timelike edges leading from $v$ to $v^{\prime}$. For example, the vertices $v$ and $v^{\prime}$ as illustrated in Figure 1 are not causally related.

The physical properties of the ensemble of causal triangulations behaves much more regular than its Euclidean counterpart. For example, it has a fractal dimension of $d_{H}=2$ instead of $d_{H}=4$ for DT. Also when coupled to simple matter models, such as the Ising model, it behaves much more like a regular lattice such as $\mathbb{Z}^{2}$, Ambjørn, Anagnostopoulos and Loll (1999).

\footnotetext{
${ }^{2}$ Recall that the fractal dimension is defined through $|B(R)| \sim R^{d_{H}}$ as $R \rightarrow \infty$, i.e. $d_{h}=$ $\lim _{R \rightarrow \infty} \log |B(R)| / \log R$.
} 
While the approach of CDT has recently lead to a number of interesting physical results, especially with respect to higher-dimensional numerical implementations (see Ambjørn et al. (2010) for a review), the probabilistic aspects of this model have hardly been studied. In fact, recently Durhuus, Jonsson and Wheater defined the uniform measure on infinite causal triangulations, cf. Durhuus, Jonsson and Wheater (2010) (see also Durhuus (2003) for earlier ideas), proving almost surely (a.s.) recurrence, that a.s. the fractal dimension is $d_{H}=2$ and that the spectral dimension is a.s. bounded from above by $d_{s} \leq 2$. A similar definition of the uniform measure has previously also been used by one of the authors of this article and M. Krikun to prove the existence of a phase transition for the Ising model coupled to CDT Krikun and Yambartsev (2012).

In this article, we extend the results by Durhuus, Jonsson and Wheater (2010) to obtain weak convergence results of the rescaled length and length-area processes. Firstly, we give a more detailed existence proof of the measure on infinite causal triangulations. Second, in the line of Angel and Schramm (2003) and Krikun (2005), we obtain novel weak convergence limits for the distribution of the area and length of the boundary of a ball of radius $t$. We see that they scale as $t^{2}$ and $t$, respectively consistent with the fractal dimension of $d_{H}=2$. These results follow from a bijection between causal triangulations and certain Galton-Watson branching processes through the size biased branching process measure Lamperti and Ney (1968) — the critical branching process distribution conditioned on survival at infinity. Exploiting the relation to conditioned critical Galton-Watson processes, one can go further and obtain weak convergence of the joint length and area process. The process is diffusive and the corresponding Kolmogorov equation enables us to derive the physical Hamiltonian, providing us with a mathematical rigorous formulation of scaling limits of CDT.

In the next section, we give basic definitions and introduce infinite causal triangulations and show existence of the measure on infinite causal triangulations in an alternative presentation to Durhuus, Jonsson and Wheater (2010). In Section 3, we then present the relation to critical Galton-Watson processes conditioned to never die out. In Section 4, we exploit this relation to obtain weak convergence of the length process (Theorem 4.1) and the joint length-area process (Theorem 4.2). Theorem 4.1 is proven in Appendix. These results provide a mathematical rigorous formulation of certain scaling limits of CDT which we discuss in Section 5.

\section{Infinite causal triangulations}

We consider rooted causal triangulations of $\mathcal{C}_{h}=S^{1} \times[0, h], h=1,2, \ldots$, and of $\mathcal{C}=S^{1} \times[0, \infty)$, where $S^{1}$ stands for a unite circle.

Let $\overline{\mathcal{C}_{h}}$ be the cylinder $\mathcal{C}_{h}$ with two open discs with radius one $O_{0}$ and $O_{h}$, such that $\mathcal{C}_{h} \cup O_{0} \cup O_{h}$ is homeomorphic to the unit sphere. The basic notion we need is embedding of a (finite) graph $G$ on the surface $\overline{\mathcal{C}_{h}}$. Informally, an embedding $i$ : 
$G \rightarrow \overline{\mathcal{C}_{h}}$ of a graph $G$ into $\overline{\mathcal{C}_{h}}$ is its representation on $\overline{\mathcal{C}_{h}}$ in such a way that its edges may intesect only at their endpoints. $\overline{\mathcal{C}_{h}}$ is homeomorphic to a two-dimensional unit sphere, thus $G$ belongs to class of planar graphs. Remember that all connected components of $\overline{\mathcal{C}_{h}} \backslash i(G)$ are homeomorphic to a two-dimensional unit ball and are called faces. Let the size of a face be the number of edges incident to it, with the convention that an edge incident to the same face on both sides counts for two. We then call a face with size 3 (or 3-sided face) a triangle.

Remark 1. Some care has to be put into the definition of what is meant by a triangle due to self-loops and multiple edges, and in particular, a simple definition like "the face is a triangle if its boundary meets precisely three edges of the graph" is not enough.

Definition 1. Consider a (finite) connected graph $G$. Its embedding $i: G \rightarrow \overline{\mathcal{C}_{h}}$ is called a causal triangulation $T$ of $\mathcal{C}_{h}$ if the following conditions hold:

- the (open) disks attached to $\mathcal{C}_{h}$ in order to get $\overline{\mathcal{C}_{h}}$ are faces of $T$, and all faces, with possible exception of these two disks, are triangles;

- each face of $T$ that belongs to $\mathcal{C}_{h}$ belongs to some strip $S^{1} \times[j, j+1], j=$ $0,1, \ldots, h-1$ and has all vertices and exactly one side on the boundary $\left(S^{1} \times\right.$ $\{j\}) \cup\left(S^{1} \times\{j+1\}\right)$ of the strip $S^{1} \times[j, j+1]$.

Definition 2. A causal triangulation $T$ of $\mathcal{C}_{h}$ is called rooted if it has a root. The root $(x, e)$ of $T$ consists of a vertex $x$ and a directed edge $e$ that runs from $x$, they are called root vertex and root edge correspondingly. The root vertex and the root edge belong to $S^{1} \times\{0\}$. The orientation induced by the ordered pair that consists of the root edge and the vector that runs from the root vertex in positive time direction coincides with the fixed orientation of $\overline{\mathcal{C}_{h}}$.

Definition 3. Two rooted causal triangulations of $\mathcal{C}^{h}$, say $T$ and $T^{\prime}$, are equivalent if the following conditions hold:

1. $T$ and $T^{\prime}$ are embeddings of the same graph $G$, that is, $T$ is embedding $i: G \rightarrow \overline{\mathcal{C}^{h}}$ and $T^{\prime}$ is embedding $j: G \rightarrow \overline{\mathcal{C}^{h}}$;

2. there exists a self-homeomorphism $\tilde{h}: \overline{\mathcal{C}^{h}} \rightarrow \overline{\mathcal{C}^{h}}$ such that $\tilde{h} i=j$. Here we suppose that $\tilde{h}$ not only takes $i(G)$ to $j(G)$ but also transforms each slice $S^{1} \times\{j\}$, $j=0, \ldots, h$ to itself and sends the root of $T$ to the root of $T^{\prime}$.

For convenience, we usually abbreviate "equivalence class of rooted causal triangulations" to "causal triangulation" or CT.

Cutting off the stripe $S^{1} \times(h, h+1]$ from $\mathcal{C}^{h+1}$ we obtain a natural map from the set of causal triangulations of $\mathcal{C}^{h+1}$ to the set of causal triangulations of $\mathcal{C}^{h}$ that we denote by $r_{h}$. 
Definition 4. We say that $T$ is a causal triangulation of $\mathcal{C}$, if $T=\left(T_{1}, T_{2}, \ldots\right)$, where $T_{h}$ is a causal triangulation of $\mathcal{C}_{h}, h=1,2, \ldots$, and the sequence is subject to consistency condition $T_{h}=r_{h}\left(T_{h+1}\right), h=1,2, \ldots$

By $\mathbb{C} \mathbb{T}_{\infty}$ denote the set of all causal triangulations of $\mathcal{C}$ and by $\mathbb{C} \mathbb{T}_{h}$ denote the set of all causal triangulations of $\mathcal{C}^{h}$. Let

$$
\mathbb{L} \mathbb{T} h=\bigcup_{i=1}^{h} \mathbb{C} \mathbb{T}_{i}, \quad h=1,2, \ldots \quad \text { and } \quad \mathbb{L} \mathbb{T} \infty=\mathbb{C} \mathbb{T}_{\infty} \cup \bigcup_{i=1}^{\infty} \mathbb{C} \mathbb{T}_{i}
$$

The restriction map $r_{h}: \mathbb{C} \mathbb{T}_{h+1} \rightarrow \mathbb{C T}_{h}$ can be naturally generalized to become the restriction map $r_{h}: \mathbb{L} \mathbb{T} \infty \rightarrow \mathbb{L} \mathbb{T} h$. We see that $T \in \mathbb{L} \mathbb{T} \infty$ is identified by the sequence $\left(T_{1}, T_{2}, \ldots\right)$, where $T_{h} \in \mathbb{L} \mathbb{T} h$ are subject only to consistency condition $T_{h}=r_{h}\left(T_{h+1}\right), h=1,2, \ldots$.

We use the standard formalism for plane trees (see Neveu (1986) or Aldous and Pitman (1998)). Let

$$
\mathscr{U}=\bigcup_{n=0}^{\infty} \mathbb{N}^{n}
$$

where $\mathbb{N}=\{1,2, \ldots\}$ and by convention $\mathbb{N}^{0}=\{\varnothing\}$. The height of $u=\left(u_{1}, \ldots\right.$, $\left.u_{n}\right) \in \mathbb{N}^{n}$ is $|u|=n$. If $u=\left(u_{1}, \ldots u_{m}\right)$ and $v=\left(v_{1}, \ldots, v_{n}\right)$ belong to $\mathscr{U}$, then $u v=\left(u_{1}, \ldots, u_{m}, v_{1}, \ldots, v_{n}\right)$ denotes the concatenation of $u$ and $v$. In particular, $u \varnothing=\varnothing u=u$. If $v$ is of the form $v=u j$ for $u \in \mathscr{U}$ and $j \in \mathbb{N}$, we say that $u$ is the predecessor of $v$, or that $v$ is a successor of $u$. More generally, if $v$ is of the form $v=u w$ for $u, w \in \mathscr{U}$, we say that $u$ is an ancestor of $v$, or that $v$ is a descendant of $u$.

Definition 5. A (finite or infinite) family tree $\tau$ is a subset of $\mathscr{U}$ such that

(i) $\varnothing \in \tau$;

(ii) if $u \in \tau$ and $u \neq \varnothing$, the predecessor of $u$ belongs to $\tau$;

(iii) for every $u \in \tau$, there exists an integer $k_{u}(\tau) \geq 0$ such that $u j \in \tau$ if and only if $1 \leq j \leq k_{u}(\tau)$.

The height of a finite tree is the maximum height of all vertices in the tree. Let $\mathbb{T}^{(\infty)}$ be the set of all family trees and $\mathbb{T}^{(h)}$ the set of all finite family trees of height at most $h$. There is a natural restriction map $r_{h}: \mathbb{T}^{(\infty)} \rightarrow \mathbb{T}^{(h)}$ such that if $\tau$ is a family tree, then $r_{h} \tau$ is the tree formed by all vertices of $\tau$ of height at most $h$. Let $\mathbb{T}_{\infty}$ be the set of all infinite family trees and $\mathbb{T}_{h}$ the set of family trees of height $h$.

A family tree $\tau \in \mathbb{T}^{(\infty)}$ is identified by the sequence $\left(r_{h} \tau, h \geq 1\right)$. Note that the $r_{h} \tau \in \mathbb{T}^{(h)}$ are subject only to the consistency condition that $r_{h} \tau=r_{h}\left(r_{h+1} \tau\right)$.

Theorem 2.1. There is a bijection $\phi: \mathbb{L} \mathbb{T} \infty \rightarrow \mathbb{T}^{(\infty)}$ such that 


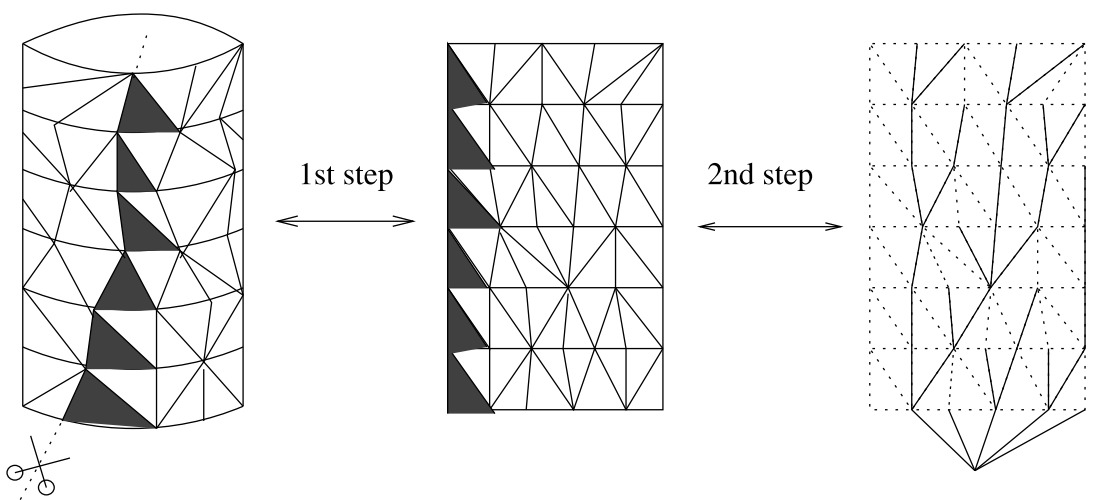

Figure 2 Tree parametrization. The following two steps outline how to go from a causal triangulation to a tree: Step 1. The first step is cutting. We construct the sequence of root vertices (or triangles) on each slice $S^{1} \times\{i\}$ by the following rule. Let $v_{1}$ be the vertex in the slice $S^{1} \times\{1\}$ which belongs to the rooted triangle containing the root edge $\left[v_{0}, v_{0}^{\prime}\right]$. One chooses the right most neighbour $v_{1}^{\prime}$ of $v_{1}$ on the slice $S^{1} \times\{1\}$ as the new rooted edge. Following this procedure we can cut open the triangulation along the left-hand side of the root triangles. Step 2. We add one vertex in the slice below the initial boundary and connect all vertices on the initial boundary to this vertex. We then remove all spatial (horizontal edges) and each leftmost outgoing time-like edge of every vertex. The lowest vertex is then connected to the root. The resulting graph is a tree. The inverse relation should now be clear from the construction.

- $\phi \circ r_{h}=r_{h} \circ \phi$, that is, $\phi$ respects $r_{h}, h=1,2, \ldots$;

- for $t=1,2, \ldots, \infty$, restrictions of $\phi$ to $\mathbb{C T}_{t}$ denoted by $\phi_{t}: \mathbb{C} \mathbb{T}_{t} \rightarrow \mathbb{T}_{t}$ are also bijections that respect $r_{h}, h=1,2, \ldots$.

This theorem dates back to Di Francesco, Guitter and Kristjansen (2001) and a detailed proof can be found in Durhuus, Jonsson and Wheater (2010) (see also Malyshev, Yambartsev and Zamyatin (2001)). We refer to Figure 2 for an illustration of the proof.

The set $\mathbb{T}^{(\infty)}$ is now identified as a subset of an infinite product of countable sets

$$
\mathbb{T}^{(\infty)} \subset \mathbb{T}^{(0)} \times \mathbb{T}^{(1)} \times \mathbb{T}^{(2)} \times \cdots
$$

We give $\mathbb{T}^{(\infty)}$ the topology derived by this identification from the product of discrete topologies on $\mathbb{T}^{(h)}$. Therefore, a sequence of family trees $\tau_{n}$ has a limit

$$
\lim \tau_{n}=\tau \in \mathbb{T}^{(\infty)}
$$

iff for every $h$ there exist a $\tau^{(h)} \in \mathbb{T}^{(h)}$ and $n(h)$ such that $r_{h} \tau_{n}=\tau^{(h)}$ for all $n \geq n(h)$; the limit is then the unique $\tau \in \mathbb{T}^{(\infty)}$ with $r_{h} \tau=\tau^{(h)}$. In particular, for each $\tau \in \mathbb{T}^{(\infty)}$ the sequence $r_{h} \tau$ has limit $\tau$ as $n \rightarrow \infty$. The topology is metrizable, for example, set $d\left(\tau, \tau^{\prime}\right)=k^{-1}$, where

$$
k=\sup \left\{h: r_{h} \tau=r_{h} \tau^{\prime}\right\} .
$$


It is easy to see that the metric space is complete and separable.

The topology gives us the Borel $\sigma$-algebra to define probability measures on it. Besides, we can define the weak convergence of measures. As usual, a measure $\mu$ is the weak limit of the sequence of measures $\mu_{n}$ if

$$
\int f d \mu_{n} \rightarrow \int f d \mu, \quad \text { as } n \rightarrow \infty
$$

for every bounded continuous real-valued function $f$ given on $\mathbb{T}^{(\infty)}$.

Let $\mathbb{T}=\bigcup_{h=0}^{\infty} \mathbb{T}^{(h)}$. Consider a system of nonnegative numbers

$$
\pi=\{p(\tau), \tau \in \mathbb{T}\}
$$

such that the following conditions hold:

1. for $h=0,1,2, \ldots$ we have

$$
\sum p\left(\tau_{h+1}\right)=p\left(\tau_{h}\right) \quad \text { for any } \tau_{h} \in \mathbb{T}^{(h)},
$$

where the sum is over $\tau_{h+1} \in \mathbb{T}^{(h+1)}$ such that $r_{h} \tau_{h+1}=\tau_{h}$;

2. $p\left(\tau_{0}\right)=1$ for $\tau_{0} \in \mathbb{T}^{(0)}$.

Note that if $\mu$ is a probability measure on $\mathbb{T}^{(\infty)}$, then

$$
\pi=\left\{\mu\left(\left\{\tau \in \mathbb{T}^{(\infty)}: r_{h} \tau=\tau_{h}\right\}\right): \tau_{h} \in \mathbb{T}^{(h)}, h=0,1,2, \ldots\right\}
$$

is a system of numbers that satisfies the above two conditions.

The following fact can easily be checked (it is proved in the same way as Kolmogorov extension theorem), and it helps to define a measure on $\mathbb{T}^{(\infty)}$. The fact is that for every system of nonnegative numbers $\pi$ satisfying the two conditions above there is a probability measure $\mu$ on $\mathbb{T}^{(\infty)}$ such that

$$
p\left(\tau_{h}\right)=\mu\left(\left\{\tau \in \mathbb{T}^{(\infty)}: r_{h} \tau=\tau_{h}\right\}\right) \quad \text { for all } \tau_{h} \in \mathbb{T}^{(h)}, h=0,1,2, \ldots
$$

In other words, a random family tree is a random element of $\mathbb{T}^{(\infty)}$, formally specified by its sequence of restrictions, say $\mathcal{T}=\left(r_{h} \mathcal{T}, h=0,1, \ldots\right)$, where each $r_{h} \mathcal{T}$ is a random variable with values in the countable set $\mathbb{T}^{(h)}$, and $r_{h} \mathcal{T}=r_{h}\left(r_{h+1} \mathcal{T}\right)$ for all $h$. The distribution of $\mathcal{T}$ is determined by the sequence of distributions of $r_{h} \mathcal{T}$ for $h \geq 0$. Such a distribution is determined by a specification of the conditional distributions of $r_{h+1} \mathcal{T}$ given $r_{h} \mathcal{T}$ for $h \geq 0$. To give a more exact specification of the distribution of $\mathcal{T}$, we need some definitions.

For every $v \in \mathscr{U}$, let $c_{v} \tau$ be the number of successors of $v$ (if $v \notin \tau$, then $\left.c_{v} \tau=0\right)$. For every $\tau \in \mathbb{T}^{(\infty)}$ and $g \geq 0$, let the $g$ th generation of individuals in $\tau$, denoted by $\operatorname{gen}(g, \tau)$, be the set of $u \in \tau$ such that the height of $u$ is $g$, also let $Z_{g} \tau$ be the number of elements of the set $\operatorname{gen}(g, \tau)$ (to simplify notation let $Z=Z_{0}$ ). Note that $\mathbb{T}^{(0)}$ contains only one family tree that consists of only one element $\varnothing$, and for any $\tau \in \mathbb{T}^{(\infty)}$, we have $r_{0} \tau=\{\varnothing\}$. A family tree $\tau$ is conveniently specified as the unique $\tau \in \mathbb{T}^{(\infty)}$ such that $r_{h} \tau=\tau^{(h)}$ for all $h$ for 
some sequence of trees $\tau^{(h)} \in \mathbb{T}^{(h)}$ determined recursively as follows. Given that $\tau^{(h)} \in \mathbb{T}^{(h)}$ has been defined $\left(\tau^{(0)}\right.$ is the unique tree from $\left.\mathbb{T}^{(h)}\right)$, the set of vertices gen $(h, \tau)=\operatorname{gen}\left(h, \tau^{(h)}\right)=r_{h} \tau \backslash r_{h-1} \tau$ is determined, hence so is the size $Z_{h} \tau=Z_{h} \tau^{(h)}$ of this set; for each possible choice of $Z_{h} \tau$ non-negative integers $\left(a_{v}, v \in \operatorname{gen}(h, \tau)\right)$, there is a unique $\tau^{(h+1)} \in \mathbb{T}^{(h+1)}$ such that $r_{h} \tau^{(h+1)}=\tau^{(h)}$ and $c_{v} \tau^{(h+1)}=a_{v}$ for all $v \in \operatorname{gen}(h, \tau)$. So a unique $\tau \in \mathbb{T}^{(\infty)}$ is determined by specifying for each $h \geq 0$ the way in which these $Z_{h} \tau$ non-negative integers are chosen given that $r_{h} \tau=\tau^{(h)}$ for some $\tau^{(h)} \in \mathbb{T}^{(h)}$.

Thus a more exact specification of the distribution of $\mathcal{T}$ is a specification of the joint conditional distribution given $r_{h} \tau$ of the numbers of children $c_{v} \tau$ as $v$ ranges over gen $(h, \tau)$ for $h \geq 0$.

Since the topology on $\mathbb{T}^{(\infty)}$ is a product of discrete topologies on $\mathbb{T}^{(h)}, h \geq$ 0 , the weak convergence of measures on $\mathbb{T}^{(\infty)}$ can be easily reformulated in the following way. For random family trees $\mathcal{T}_{n}, n=1,2, \ldots$ and $\mathcal{T}$, we say that $\mathcal{T}_{n}$ converges in distribution to $\mathcal{T}$, and write $\operatorname{dist}\left(\mathcal{T}_{n}\right) \rightarrow \operatorname{dist}(\mathcal{T})$ if

$$
\mathbb{P}\left(r_{h} \mathcal{T}_{n}=\tau\right) \rightarrow \mathbb{P}\left(r_{h} \mathcal{T}=\tau\right) \quad \forall h \geq 0, \tau \in \mathbb{T} .
$$

\section{Infinite causal triangulations and critical branching processes}

Let $p(\cdot)=(p(0), p(1), \ldots)$ be a probability distribution on the non-negative integers with $p(1)<1$. Call a random family tree $\mathcal{G}$ a Galton-Watson $(G W)$ tree with offspring distribution $p(\cdot)$ if the number of children $Z \mathcal{G}$ of the root has distribution $p(\cdot)$ :

$$
\mathbb{P}(Z \mathcal{G}=n)=p(n) \quad \forall n \geq 0
$$

and for each $h=1,2, \ldots$, conditionally given $r_{h} \mathcal{G}=t^{(h)}$, the numbers of children $c_{v} \mathcal{G}, v \in \operatorname{gen}\left(h, t^{(h)}\right)$, are i.i.d. according to $p(\cdot)$.

Introduce the generating function $f(s)=\sum_{n \geq 0} p(n) s^{n}$. We consider only the critical GW process which has $f^{\prime}(1)=1$. Suppose further that $v=f^{\prime \prime}(1) / 2<\infty$. A random family tree $\mathcal{G}^{\infty}$, which we call $\mathcal{G}$ conditioned on non-extinction is derived from the family tree $\mathcal{G}$ in the way described in Theorem 3.1 below. The probabilistic description of $\mathcal{G}^{\infty}$ involves the size-biased distribution $p^{*}(\cdot)$ associated with probability distribution $p(\cdot)$ :

$$
p^{*}(n)=n p(n) \quad \forall n \geq 0 .
$$

Putting together Proposition 2 and Proposition 5 from Aldous and Pitman (1998) (they correspond to reformulation in this family tree language of theorems from Kesten (1986) and Kennedy (1975)), we have the following theorem. 


\section{Theorem 3.1.}

1. Let $\# \mathcal{G}$ be the number of vertices in the tree $\mathcal{G}$. Then

$$
\operatorname{dist}(\mathcal{G} \mid \# \mathcal{G}=n) \rightarrow \operatorname{dist}\left(\mathcal{G}^{\infty}\right) \quad \text { as } n \rightarrow \infty,
$$

where $\operatorname{dist}\left(\mathcal{G}^{\infty}\right)$ is the distribution of a random family tree $\mathcal{G}^{\infty}$ specified by

$$
\mathbb{P}\left(r_{h} \mathcal{G}^{\infty}=\tau\right)=Z_{h} \tau \mathbb{P}\left(r_{h} \mathcal{G}=\tau\right) \quad \forall \tau \in \mathbb{T}^{(h)}, h \geq 0 .
$$

2. Almost surely $\mathcal{G}^{\infty}$ contains a unique infinite path $\left(V_{0}, V_{1}, V_{2}, \ldots\right)$ such that $V_{0}=\varnothing$ and $V_{h+1}$ is a successor of $V_{h}$ for every $h=0,1,2, \ldots$

3. For each $h$ the joint distribution of $r_{h} \mathcal{G}^{\infty}$ and $V_{h}$ is given by

$$
\mathbb{P}\left(r_{h} \mathcal{G}^{\infty}=\tau, V_{h}=v\right)=\mathbb{P}\left(r_{h} \mathcal{G}=\tau\right) \quad \forall \tau \in \mathbb{T}^{(h)}, v \in \operatorname{gen}(h, \tau), h \geq 0 .
$$

4. The joint distribution of $\left(V_{0}, V_{1}, V_{2}, \ldots\right)$ and $\mathcal{G}^{\infty}$ is determined recursively as follows: for each $h \geq 0$, given $\left(V_{0}, V_{1}, \ldots, V_{h}\right)$ and $r_{h} \mathcal{G}^{\infty}$, the numbers of successors $c_{v} \mathcal{G}^{\infty}$ are independent as $v$ ranges over gen $\left(h, \mathcal{G}^{\infty}\right)$, with distribution $p(\cdot)$ for $v \neq V_{h}$, and with the size-biased distribution $p^{*}(\cdot)$ for $v=V_{h}$; given also the numbers of successors $c_{v} \mathcal{G}^{\infty}$ for $v \in \operatorname{gen}\left(h, \mathcal{G}^{\infty}\right)$, the vertex $V_{h+1}$ has uniform distribution on the set of $c_{V_{h}} \mathcal{G}^{\infty}$ successors of $V_{h}$.

Remark 2. The UICT (Uniform Infinite Causal Triangulation) is a special case for which the critical branching process has off-spring probability $p(n)=(1 / 2)^{n+1}$. In this case the conditional probability of the left-hand side of (3.1) provides the same probability for any tree as well as CT with $n$ vertices and thus defines the uniform measure on this set. The measure on the right-hand side of (3.1) determines the uniform measure on the set of infinite causal triangulation (UICT).

Remark 3. Another measure of interest is the Gibbs measure on the set of CTs. Its Hamiltonian $H$ is simply the number of triangles multiplied by a coupling $\tilde{\lambda}$ (the "bare" cosmological constant). There is a correspondence between the number of triangles and vertices in a tree: let $\tau^{(h)}$ be some finite tree of height $h$ and $t^{(h)}$ its corresponding causal triangulation. The number of triangles in $t^{(h)}$ is equal to $1+2 \sum_{k=1}^{h-1} Z_{k} \tau^{(h)}+Z_{h} \tau^{(h)}=H\left(t^{(h)}\right)$. The probability of $t^{(h)}$ on the set of casual triangulations of the "disc" with height $h$ is given by the Gibbs measure $P_{h}\left(t^{(h)}\right)=Z_{h}^{-1} e^{-\tilde{\lambda} H\left(t^{(h)}\right)}$, where $Z_{h}^{-1}$ is the normalisation. Moreover, it is not difficult to prove that for $\tilde{\lambda}=\ln 2$ the measure $P_{h}$ also converges to the UICT as $h \rightarrow \infty$.

\section{Weak convergence from conditioned critical branching processes}

Having established the relation between infinite causal triangulations and critical Galton-Watson processes conditioned to never die out in the previous section, one 
can now use several known convergence results for the conditioned branching process to determine the corresponding convergence of several observables.

From the point of view of universality one expects that the continuum processes shall be the same for any kind of underlying critical Galton-Watson process. We will see that this is indeed the case. Let us therefore consider an arbitrary critical Galton-Watson process $\mathcal{G}$ with generating function $f(s)=\sum_{n \geq 0} p(n) s^{n}$ of the off-spring distribution $p(\cdot)$. Since the process is critical we have $f^{\prime}(1)=1$. Let us further assume that $v=f^{\prime \prime}(1) / 2<\infty$. For short hand denote the size of the $t$ 's generation by $\eta_{t} \equiv Z_{t} \mathcal{G}$. It was shown by Lindvall $(1972,1974)$ that if $\eta_{0}=$ $v t x+o(t)$ with $x>0$ :

$$
\frac{\eta_{[t \tau]}}{v t} \Rightarrow X_{\tau}, \quad 0 \leq \tau<\infty,
$$

where $\Rightarrow$ denotes weak convergence on the functions space $D[0, \infty)$ and the continuous process solves the following Itô's equation

$$
d X_{\tau}=\sqrt{2 X_{\tau}} d B_{\tau}, \quad X_{0}=x,
$$

with $B_{\tau}$ standard Brownian motion of variance 1 .

Let us note that the finite-dimensional distributions of $\eta_{t}$ can be easily obtained from the following relation due to Kesten, Ney and Spitzer for the generating function of the size of the $t$ 's generation of a critical Galton-Watson process with $v=f^{\prime \prime}(1) / 2<\infty$ and $\eta_{0}=1$ (e.g., see, Athreya and Ney (1972))

$$
\frac{1}{1-f_{t}(s)}=\frac{1}{1-s}+v t+o(t), \quad \text { uniformly for } 0 \leq s<1 \text {. }
$$

Tightness can then be obtained by standard techniques (e.g., see Billingsley (1999)). An alternative detailed proof of Lindvall's theorem using convergence of the generator of the Markov process can be found in Ethier and Kurtz (1986).

We now investigate the convergence of the length of the boundary of an infinite CT as a process of time. Since any Galton-Watson tree conditioned to never die out is in bijection with an infinite $\mathrm{CT}$, we refer to the corresponding probability measure as an infinite CT constructed from a critical Galton-Watson process. The UICT is a special case for which the critical branching process has off-spring probability $p(n)=(1 / 2)^{n+1}$. In particular, this off-spring probability satisfies $f^{(n)}(1)<\infty$ for all $n \in \mathbb{N}$.

By the relation discussed in the previous section, the size of the boundary $k_{t}$ of an infinite CT constructed from a critical Galton-Watson process at time $t$ corresponds to the size of the $t$ 's generation of the Galton-Watson process conditioned to never die out, denoted by $\hat{\eta}_{t} \equiv Z_{t} \mathcal{G}^{\infty}$. Define the length process

$$
k_{\tau}^{(t)}:=\frac{k_{[t \tau]}}{\nu t} \equiv \frac{\hat{\eta}_{[t \tau]}}{\nu t}, \quad 0 \leq \tau<\infty .
$$

The convergence of the finite-dimensional distributions of the process $\left\{\hat{\eta}_{t}\right\}$ was studied in Lamperti and Ney (1968), and we can deduce the following theorem for the length process (4.1): 
Theorem 4.1. For an infinite CT constructed from a critical Galton-Watson process with $v=f^{\prime \prime}(1) / 2<\infty$ and $f^{\prime \prime \prime}(1)<\infty$, and initial boundary $m_{0} \equiv k_{0}=$ $v t l+o(t)$ with $l \geq 0$ we have

$$
k_{\tau}^{(t)} \Rightarrow L_{\tau}, \quad 0 \leq \tau<\infty
$$

in the sense of weak convergence on the functions space $D[0, \infty)$, where the continuous process solves the following Itô's equation

$$
d L_{\tau}=2 d \tau+\sqrt{2 L_{\tau}} d B_{\tau} \quad L_{0}=\ell
$$

The process $L_{\tau}$ is diffusive and the Feynman-Kac equation for $\phi_{\xi}(\ell, \tau)=$ $\mathbb{E}\left[\exp \left(-\xi L_{\tau}\right) \mid L_{0}=\ell\right]$ is given by

$$
-\frac{\partial}{\partial \tau} \phi_{\xi}(\ell, \tau)=\hat{H} \phi_{\xi}(\ell, \tau), \quad \hat{H}=-2 \frac{\partial}{\partial \ell}-\ell \frac{\partial^{2}}{\partial \ell^{2}}, \quad \phi_{\xi}(\ell, 0)=e^{-\xi \ell} .
$$

Here the operator $\hat{H}$ is known in the physics literature as the Hamiltonian of two-dimensional CDT (having cosmological constant equal zero, see, for example, Ambjørn and Loll (1998)).

In order to prove the convergence of the process one also has to prove tightness. The complete proof of Theorem 4.1 is presented in the Appendix.

Corollary 4.1. In the special case of $l=0$, corresponding to an infinite CT constructed from a critical Galton-Watson process with zero initial boundary, we have

$$
\mathbb{E}\left[e^{-\xi L_{\tau}} \mid L_{0}=0\right]=\frac{1}{(1+\xi \tau)^{2}}
$$

which for $\tau=1$ is a random variable with gamma distribution with parameter two, that is, $\mathbb{P}\left(L_{1} \in d x\right) / d x=x e^{-x}, x \geq 0$. (The sum of two independent random variables with exponential distribution with rate 1 .)

This gives the distribution of the rescaled upper boundary $L_{1}$, i.e. of the random variable $k_{t} / t$ in the limit $t \rightarrow \infty$. It is hence the analog of Theorem 4 of Krikun (2005) which states the corresponding result for uniform infinite planar triangulations.

We now want to discuss the convergence of the rescaled area of a neighbourhood $\Gamma_{t}$ with time-radius $t$. Let us denote the number of triangles in $\Gamma_{t}$ by $\alpha_{t}$ and define the area process as

$$
\alpha_{\tau}^{(t)}:=\frac{\alpha_{[t \tau]}}{\nu t^{2}}, \quad 0 \leq \tau<\infty .
$$

We then have the following theorem based on a theorem of Pakes for conditioned critical Galton-Watson processes Pakes (1999): 
Theorem 4.2. For an infinite CT constructed from a critical Galton-Watson process with $v=f^{\prime \prime}(1) / 2<\infty$ and $f^{\prime \prime \prime}(1)<\infty$, and initial boundary $m_{0} \equiv k_{0}=$ $v t l+o(t)$ we have

$$
\left(k_{\tau}^{(t)}, \alpha_{\tau}^{(t)}\right) \Rightarrow\left(L_{\tau}, 2 \int_{0}^{\tau} L_{u} d u\right), \quad 0 \leq \tau<\infty,
$$

in the sense of weak convergence on the functions space $D[0, \infty) \times D[0, \infty)$, where the continuous process $L_{\tau}$ solves the Itô's equation as in Theorem 4.1

$$
d L_{\tau}=2 d \tau+\sqrt{2 L_{\tau}} d B_{\tau} \quad L_{0}=l .
$$

The Feynman-Kac equation for $\phi_{\xi, \lambda}(l, \tau)=\mathbb{E}\left[\exp \left(-\xi L_{\tau}-2 \lambda \int_{0}^{\tau} L_{u} d u\right) \mid L_{0}=l\right]$ is given by

$$
\begin{aligned}
-\frac{\partial}{\partial \tau} \phi_{\xi, \lambda}(l, \tau) & =\hat{H} \phi_{\xi, \lambda}(l, \tau), \\
\hat{H} & =-2 \frac{\partial}{\partial l}-l \frac{\partial^{2}}{\partial l^{2}}+2 \lambda l, \quad \phi_{\xi, \lambda}(l, 0)=e^{-\xi l} .
\end{aligned}
$$

Proof. By construction of the bijection between CTs and Galton-Watson trees we have $\alpha_{t}=k_{0}+2\left(k_{1}+\cdots+k_{t-1}\right)+k_{t}$, that is, each internal spatial (horizontal) edge is connected to two triangles while each boundary edge is connected to one triangle (see Figure 1). Hence,

$$
\alpha_{\tau}^{(t)}=\frac{\alpha_{[t \tau]}}{v t^{2}}=\frac{1}{v t^{2}}\left(2 \sum_{i=0}^{[t \tau]} \hat{\eta}_{i}-\hat{\eta}_{0}-\hat{\eta}_{[t \tau]}\right)=2 \int_{0}^{\tau} k_{u}^{(t)} d u+o(1) .
$$

Following ideas of Pakes (1999), Theorem 3.3, the weak convergence of $\left(k_{\tau}^{(t)}, \alpha_{\tau}^{(t)}\right)$ then follows from the weak convergence of

$$
\left(k_{\tau}^{(t)}, 2 \int_{0}^{\tau} k_{u}^{(t)}\right) \Rightarrow\left(L_{\tau}, 2 \int_{0}^{\tau} L_{u} d u\right) .
$$

It is enough to note that by (4.3) we have that $h\left(k_{\tau}^{(t)}\right):=\left(k_{\tau}^{(t)}, \alpha_{\tau}^{(t)}\right)$ is a continuous functional of $k_{\tau}^{(t)}$ and hence the convergence of $\left(k_{\tau}^{(t)}, \alpha_{\tau}^{(t)}\right) \Rightarrow\left(L_{\tau}, 2 \int_{0}^{\tau} L_{u} d u\right)$ follows by the continuous mapping theorem (Theorem 2.7, Billingsley (1999)) applied to Theorem 4.1.

Having established the convergence, we can then apply the Feynman-Kac formula to

$$
\phi_{\xi, \lambda}(l, \tau)=\mathbb{E}\left[e^{-\xi L_{\tau}-2 \lambda \int_{0}^{\tau} L_{u} d u} \mid L_{0}=l\right]
$$

with

$$
d L_{\tau}=2 d t+\sqrt{2 L_{\tau}} d B_{\tau} \quad L_{0}=l
$$


which yields

$$
-\frac{\partial}{\partial \tau} \phi_{\xi, \lambda}(l, \tau)=\left(-2 \frac{\partial}{\partial l}-l \frac{\partial^{2}}{\partial l^{2}}+2 \lambda l\right) \phi_{\xi, \lambda}(l, \tau), \quad \phi_{\xi, \lambda}(l, 0)=e^{-\xi l} .
$$

The last equation is again known from the physics literature in the context of CDT with cosmological constant $\lambda$. In fact, one can easily solve the differential equation leading to

$$
\phi_{\xi, \lambda}(l, \tau)=\frac{\bar{\xi}^{2}(\xi, \tau)-2 \lambda}{\xi^{2}-2 \lambda} e^{-l \bar{\xi}(\xi, \tau)}
$$

with

$$
\bar{\xi}(\xi, \tau)=\sqrt{2 \lambda} \operatorname{coth}(\sqrt{2 \lambda} \tau)-\frac{2 \lambda}{\sinh ^{2}(\sqrt{2 \lambda} \tau)[\xi+\sqrt{2 \lambda} \operatorname{coth}(\sqrt{2 \lambda} \tau)]} .
$$

Corollary 4.2. Setting $\tau=1$ and $l=0$ in (4.4) one has

$$
\phi_{\xi, \lambda}(0,1)=\frac{2 \lambda}{(\sqrt{2 \lambda} \cosh \sqrt{2 \lambda}+\xi \sinh \sqrt{2 \lambda})^{2}}
$$

which also follows from Pakes (1999), Theorem 3.3. In particular, for $\lambda=0$ one recovers

$$
\mathbb{E}\left[e^{-\xi L_{1}} \mid L_{0}=0\right]=\frac{1}{(1+\xi)^{2}}
$$

as in Corollary 4.1 and for $\xi=0$

$$
\mathbb{E}\left[e^{-\lambda A_{1}} \mid L_{0}=0\right]=\frac{1}{\cosh ^{2}(\sqrt{2 \lambda})}
$$

with $A_{1}=2 \int_{0}^{1} L_{u} d u$.

This gives the distribution of the random variable $A_{1}$, that is, $\alpha_{t} / t^{2}$ in the limit $t \rightarrow \infty$. The distribution of $A_{1}$ appears at several places related to the study of Brownian motion as has been exposed for example, in Biane, Pitman and Yor (2001) and based on their discussion we can make two remarks:

Remark 4. The random variable $A_{1}$, as introduced in Remark 4.2, can be written in the following series representation

$$
A_{1}=\frac{2}{\pi^{2}} \sum_{n=1}^{\infty} \frac{\Gamma_{n}}{(n-1 / 2)^{2}},
$$

where the $\Gamma_{n}, n \geq 1$ are i.i.d. random variables with gamma distribution with parameter two, that is, $\mathbb{P}\left(\Gamma_{n} \in d x\right) / d x=x e^{-x}, x \geq 0$. The relation can easily be 
seen by noting that

$$
\mathbb{E}\left(e^{-\lambda \Gamma_{n}}\right)=\frac{1}{(1+\lambda)^{2}} \quad \text { and } \quad \cosh z=\prod_{n \geq 1}\left(1+\frac{z^{2}}{\pi^{2}(n-1 / 2)^{2}}\right) .
$$

Remark 5. In the framework of Lévy-Khintchine representations a distribution is called infinitely divisible iff its Laplace transform $\phi(\lambda)$ admits the following representation

$$
\phi(\lambda)=\exp \left(-c \lambda-\int_{0}^{\infty}\left(1-e^{-\lambda x}\right) v(d x)\right)
$$

for some $c \geq 0$. Here, $v(d x)$ is the so-called Lévy measure and for the present application it is sufficient to consider the form of a simple density $v(d x)=\rho(x) d x$. By a straightforward and explicit computation using (4.5) and (4.6) one sees that the distribution of $A_{1}$ is infinitely divisible and has a Lévy-Khintchine representations with $c=0$ and Lévy density

$$
\rho(x)=\frac{2}{x} \sum_{n \geq 1} e^{-\pi^{2}(n-1 / 2)^{2} x / 2} .
$$

\section{Discussion}

We obtain weak convergence results for infinite causal triangulations extending the work of Durhuus, Jonsson and Wheater (2010). Under this measure the probability of a causal triangulation of a cylinder is related to a critical Galton-Watson process conditioned to never die out. We use this relation to prove novel weak convergence of the joint rescaled length-area process $\left(k_{\tau}^{(t)}, \alpha_{\tau}^{(t)}\right)$ of an infinite CT constructed from an arbitrary critical Galton-Watson process to a limiting diffusion process ( $L_{\tau}, A_{\tau}$ ), with $A_{\tau}=2 \int_{0}^{\tau} L_{u} d u$, where the Itô's equation for $L_{\tau}$ is given by (e.g., Theorems 4.1 and 4.2)

$$
d L_{\tau}=2 d \tau+\sqrt{2 L_{\tau}} d B_{\tau}, \quad L_{0}=l .
$$

In particular, we show that the Feynman-Kac formula for $\mathbb{E}\left[\exp \left(-\xi L_{\tau}-\right.\right.$ $\left.\left.\lambda A_{\tau}\right) \mid L_{0}=l\right]$ corresponds to a imaginary time Schrödinger equation with the following Hamiltonian

$$
\hat{H}\left(l, \partial_{l}\right)=-2 \frac{\partial}{\partial l}-l \frac{\partial^{2}}{\partial l^{2}}+2 \lambda l .
$$

This is the well-known Hamiltonian for two-dimensional CDT with cosmological constant $\lambda$ (see Ambjørn and Loll (1998)). ${ }^{3}$

\footnotetext{
${ }^{3}$ In fact, it is the Hamiltonian acting on an non-rooted boundary. This is due to the fact that by the construction of the Feynman-Kac or Kolmogorov backwards equation we are acting on the upper, non-rooted boundary. Alternatively, one could have also used the Kolmogorov forward equation to obtain the Hamiltonian acting on the rooted, lower boundary.
} 
By calculating the inverse Laplace transform of (4.4) one can also obtain the transition amplitude or Green's function

$$
\begin{aligned}
\phi_{\lambda}\left(l_{1}, l_{2}, \tau\right) & =\mathbb{E}\left[\mathbb{I}\left\{L_{\tau} \in d l_{2}\right\} \cdot e^{-2 \lambda \int_{0}^{\tau} L_{u} d u} \mid L_{0}=l_{1}\right] / d l_{2} \\
& =\frac{\sqrt{2 \lambda} l_{2}}{\sqrt{l_{1} l_{2}}} \frac{e^{-\sqrt{2 \lambda}\left(l_{1}+l_{2}\right) \operatorname{coth}(\sqrt{2 \lambda} \tau)}}{\sinh (\sqrt{2 \lambda} \tau)} I_{1}\left(\frac{2 \sqrt{\lambda l_{1} l_{2}}}{\sinh (\sqrt{2 \lambda} \tau)}\right),
\end{aligned}
$$

where $\mathbb{I}\{\cdot\}$ is the indicator function and $I_{1}(\cdot)$ is the modified Bessel function of first kind. This expression is also known in physics as the CDT propagator. In particular, setting $\lambda=0$ one obtains the transition amplitude for the length process

$$
\phi_{0}\left(l_{1}, l_{2}, \tau\right)=\frac{l_{2}}{\tau \sqrt{l_{1} l_{2}}} e^{-\frac{l_{1}+l_{2}}{\tau}} I_{1}\left(\frac{2 \sqrt{l_{1} l_{2}}}{\tau}\right) .
$$

This is related to the fact that if one transforms $L_{t}$ as given above to a new process $Z_{\tau}$ with $L_{\tau}=Z_{\tau}^{2} / 2$, then $Z_{\tau}$ is a Bessel process.

In conclusion, Theorems 4.1 and 4.2 provide us with a mathematical rigorous proof of certain scaling limits of two-dimensional causal dynamical triangulations (CDT). In the work Sisko, Yambartsev and Zohren (2013), we show how to obtain these results in a slightly different manner from a certain growth process of UICT. While in this article we gave a mathematical rigorous derivation for several correlations functions of CDT from the infinite causal triangulation, it would be interesting to obtain the full scaling limit using a framework like in Le Gall and Miermont (2011) work on the Brownian map in the context of DT.

\section{Appendix: Proof of Theorem 4.1}

Define $v=f^{\prime \prime}(1) / 2<\infty$ as before and $\mu=f^{\prime \prime \prime}(1) / 2<\infty$. Recall that we want to show convergence of

$$
k_{\tau}^{(t)}=\frac{\hat{\eta}_{[t \tau]}}{v t} \Rightarrow L_{\tau}, \quad 0 \leq \tau<\infty
$$

on the functions space $D[0, \infty)$. To do so, we consider the rescaled process

$$
\tilde{k}_{\tau}^{(t)}=v k_{\tau}^{(t)} \Rightarrow \tilde{L}_{\tau}=v L_{\tau}
$$

where then $\tilde{L}_{\tau}$ is a diffusion process with generator

$$
A g(x)=2 v g^{\prime}(x)+v x g^{\prime \prime}(x),
$$

where by Theorem 2.1 of Chapter 8 of Ethier and Kurtz (1986) one has $g \in$ $C_{c}^{\infty}([0, \infty))$, that is, the set of continuous functions $f:[0, \infty) \rightarrow \mathbb{R}$ which are infinitely differentiable and have compact support in $[0, \infty)$. To show convergence of the process $\tilde{k}_{\tau}^{(t)}$ to the diffusion $\tilde{L}_{\tau}$ with the above generator, we follow closely 
the strategy employed in Theorem 1.3 of Chapter 9 in Ethier and Kurtz (1986) to prove Lindvall's theorem.

Note that $\hat{\eta}_{n} / t$ is a Markov chain taking values in $E_{t}=\{l / t \mid l=1,2,3, \ldots\}$. Given $\hat{\eta}_{n}=t x$ we can then write

$$
\hat{\eta}_{n+1}=\sum_{k=1}^{t x-1} \xi_{k}+\xi_{0},
$$

where $\xi_{k}$ for $k \geq 0$ are i.i.d. random variables with generating function $f(s)$ and $\xi_{0}$ is a random variable with generating functions $s f^{\prime}(s)$. Recall that $f(s)$ is the generating function of the off-spring probabilities. The above statement follows directly from Theorem 3.1. Indeed, by Theorem 3.1 we have

$$
\sum_{k \geq 0} \mathbb{P}\left(\hat{\eta}_{n+1}=k \mid \hat{\eta}_{n}=t x\right) s^{k}=\frac{1}{t x} s \frac{d}{d s} f^{t x}(s)=f^{t x-1}(s) \cdot s f^{\prime}(s)
$$

which is the generating function for (A.4). We have

$$
\begin{aligned}
& \mathbb{E} \xi_{k}=1, \quad \mathbb{E} \xi_{k}^{2}=1+2 v, \quad \text { for } k \geq 1, \\
& \mathbb{E} \xi_{0}=1+2 v, \quad \mathbb{E} \xi_{0}^{2}=1+6 v+2 \mu .
\end{aligned}
$$

We now define

$$
T_{t} g(x)=\mathbb{E}\left\{g\left(\frac{1}{t}\left[\sum_{k=1}^{t x-1} \xi_{k}+\xi_{0}\right]\right)\right\} .
$$

By Theorem 6.5 of Chapter 1 and Corollary 8.9 of Chapter 4 of Ethier and Kurtz (1986), to prove the convergence (A.2) it is enough to show that

$$
\begin{aligned}
\lim _{t \rightarrow \infty} \sup _{x \in E_{t}} \mid t\left(T_{t} g(x)-g(x)\right) & \\
& -2 v g^{\prime}(x)-v x g^{\prime \prime}(x) \mid=0, \quad g \in C_{c}^{\infty}([0, \infty)) .
\end{aligned}
$$

For $x \in E_{t}$ we define

$$
\begin{aligned}
\varepsilon_{t}(x)= & t\left(T_{t} g(x)-g(x)\right)-2 v g^{\prime}(x)-v x g^{\prime \prime}(x) \\
= & \mathbb{E}\left\{\operatorname{tg}\left(\frac{1}{t}\left[\sum_{k=1}^{t x-1} \xi_{k}+\xi_{0}\right]\right)-\operatorname{tg}(x)-\left(\xi_{0}-1\right) g^{\prime}(x)\right. \\
& \left.-\frac{1}{2} g^{\prime \prime}(x) \frac{1}{t}\left[\left(\sum_{k=1}^{t x-1}\left(\xi_{k}-1\right)\right)^{2}+\xi_{0}-1\right]\right\} \\
= & \Delta_{t}^{1}(x)+\Delta_{t}^{2}(x),
\end{aligned}
$$


where

$$
\begin{aligned}
& \Delta_{t}^{1}(x)=\frac{1}{t} \mu g^{\prime \prime}(x) \\
& \Delta_{t}^{2}(x)=\mathbb{E}\left\{\int_{0}^{1} S_{t x}^{2} x(1-u)\left[g^{\prime \prime}\left(x+u \sqrt{\frac{x}{t}} S_{t x}\right)-g^{\prime \prime}(x)\right] d u\right\}
\end{aligned}
$$

and

$$
S_{t x}=\frac{1}{\sqrt{t x}}\left(\sum_{k=1}^{t x-1}\left(\xi_{k}-1\right)+\left(\xi_{0}-1\right)\right) .
$$

Now, since $\mu=f^{\prime \prime \prime}(1) / 2<\infty$, we have

$$
\lim _{t \rightarrow \infty} \sup _{x \in E_{t}}\left|\Delta_{t}^{1}(x)\right| \leq \mu\left\|g^{\prime \prime}\right\| \lim _{t \rightarrow \infty} \frac{1}{t}=0 .
$$

Let us suppose that $g$ has support in $[0, c]$. Then, since $\xi_{k} \geq 0$ for $k \geq 1$ and $\xi_{0} \geq 1$ we have

$$
x+u \sqrt{\frac{x}{t}} S_{t x} \geq x(1-u)
$$

hence one gets that

$$
\begin{aligned}
& \left|\int_{0}^{1} S_{t x}^{2} x(1-u)\left[g^{\prime \prime}\left(x+u \sqrt{\frac{x}{t}} S_{t x}\right)-g^{\prime \prime}(x)\right] d u\right| \\
& \quad \leq 2 x S_{t x}^{2} \int_{0 \vee(1-c / x)}^{1}(1-u)\left\|g^{\prime \prime}\right\| d u=x\left\|g^{\prime \prime}\right\|((c / x) \wedge 1)^{2} S_{t x}^{2} .
\end{aligned}
$$

To show that $\lim _{t \rightarrow \infty} \sup _{x \in E_{t}}\left|\Delta_{t}^{2}(x)\right|=0$ it suffices to show that one has $\lim _{t \rightarrow \infty}\left|\Delta_{t}^{2}\left(x_{t}\right)\right|=0$ for any convergent series $x_{t}$, as well as for $x_{t} \rightarrow 0$ and $x_{t} \rightarrow \infty$. Let us first treat the special cases $\lim _{t \rightarrow \infty} x_{t}=0$ and $\lim _{t \rightarrow \infty} x_{t}=\infty$. Note that

$$
\mathbb{E} S_{t x}^{2}=2 v+\frac{2 \mu}{t x} \leq 2(v+\mu), \quad \text { for all } t \text { and } x \in E_{t} .
$$

From (A.15) and (A.16), it then follows that $\lim _{t \rightarrow \infty}\left|\Delta_{t}^{2}\left(x_{t}\right)\right|=0$ if $\lim _{t \rightarrow \infty} x_{t}=$ 0 or $\lim _{t \rightarrow \infty} x_{t}=\infty$.

We now consider the case $\lim _{t \rightarrow \infty} x_{t}=x$, where $0<x<\infty$. In this case, one has

$$
\lim _{t \rightarrow \infty} \mathbb{E} e^{r S_{t x_{t}}}=e^{v r^{2}}
$$

and hence $S_{t x_{t}} \Rightarrow \Sigma$ with $\Sigma \sim \mathcal{N}(0,2 v)$. Following the steps of Theorem 1.3 in Ethier and Kurtz (1986) Chapter 9, one then obtains $\lim _{t \rightarrow \infty}\left|\Delta_{t}^{2}\left(x_{t}\right)\right|=0$ from (A.15) and the dominant convergence theorem. 
Hence, we showed that

$$
\lim _{t \rightarrow \infty} \sup _{x \in E_{t}}\left|\varepsilon_{t}(x)\right|=0 .
$$

Noting that the initial condition converges $\tilde{k}_{0}^{t} \rightarrow v l$ with $l \geq 0$ one completes the proof.

\section{Acknowledgments}

The authors would like to thank the anonymous referee, as well as Thordur Jonsson and Sigurdur Stefánsson for comments on improvements of the manuscript. Financial support by FAPESP under project 2010/05891-2 is acknowledged. The work of V. Sisko was supported by FAPERJ (grants E-26/170.008/2008 and E-26/110.982/2008) and CNPq (grants 471891/2006-1, 309397/2008-1 and 471946/2008-7). The work of A. Yambartsev was partly supported by CNPq 308510/2010-0. S. Zohren is currently funded by Lockheed Martin, Nokia and the University of Oxford.

\section{References}

Aldous, D. and Pitman, J. (1998). Tree-valued Markov chains derived from Galton-Watson processes. Ann. Inst. H. Poincaré Probab. Statist. 34, 637-686. MR1641670

Ambjørn, J., Anagnostopoulos, K. N. and Loll, R. (1999). A new perspective on matter coupling in 2d quantum gravity. Phys. Rev. D 60, 104035. Available at arXiv:hep-th/9904012. MR1757636

Ambjørn, J., Durhuus, B. and Jonsson, T. (1997). Quantum Geometry. A Statistical Field Theory Approach. No. 1. Cambridge Monogr. Math. Phys. Cambridge, UK: Cambridge University Press. MR1465433

Ambjørn, J., Görlich, A., Jurkiewicz, J. and Loll, R. (2010). CDT—an entropic theory of quantum gravity. ArXiv preprint arXiv:hep-th/1007.2560.

Ambjørn, J. and Loll, R. (1998). Non-perturbative Lorentzian quantum gravity, causality and topology change. Nuclear Phys. B 536, 407-434. Available at arXiv:hep-th/9805108. MR1666741

Angel, O. (2003). Growth and percolation on the uniform infinite planar triangulation. Geom. Funct. Anal. 13, 935-974. MR2024412

Angel, O. and Schramm, O. (2003). Uniform infinite planar triangulations. Comm. Math. Phys. 241, 191-213. Available at arXiv:math/0207153.

Athreya, K. and Ney, P. (1972). Branching Processes. Berlin: Springer. MR0373040

Biane, P., Pitman, J. and Yor, M. (2001). Probability laws related to the Jacobi theta and Riemann zeta functions, and Brownian excursions. Bull. Amer. Math. Soc. 38, 435-465. MR1848256

Billingsley, P. (1999). Convergence of Probability Measures, 2nd ed. Wiley Series in Probability and Statistics: Probability and Statistics. New York: John Wiley \& Sons, Inc. MR1700749

Di Francesco, P., Guitter, E. and Kristjansen, C. (2001). Generalized Lorentzian triangulations and the Calogero Hamiltonian. Nuclear Phys. B 608, 485-526. Available at arXiv:hep-th/0010259. MR1846210

Durhuus, B. (2003). Probabilistic aspects of infinite trees and surfaces. Acta Phys. Pol. 34, 47954811. 
Durhuus, B., Jonsson, T. and Wheater, J. F. (2010). On the spectral dimension of causal triangulations. J. Stat. Phys. 139, 859-881. Available at arXiv:0908.3643. MR2639893

Ethier, S. and Kurtz, T. (1986). Markov Processes: Characterization and Convergence. New York: Wiley.

Kennedy, D. P. (1975). The Galton-Watson process conditioned on the total progeny. J. Appl. Probab. 12, 800-806. MR0386042

Kesten, H. (1986). Subdiffusive behavior of random walk on a random cluster. Ann. Inst. H. Poincaré Probab. Statist. 22, 425-487. MR0871905

Krikun, M. (2005). Uniform infinite planar triangulation and related time-reversed critical branching process. J. Math. Sci. 131, 5520-5537. MR2050691

Krikun, M. and Yambartsev, A. (2012). Phase transition for the Ising model on the critical Lorentzian triangulation. J. Stat. Phys. 148, 422-439. Available at arXiv:0810.2182. MR2969626

Lamperti, J. and Ney, P. (1968). Conditioned branching processes and their limiting diffusions. Theory Probab. Appl. 12, 128-139. MR0228073

Le Gall, J.-F. and Miermont, G. (2011). Scaling limits of random trees and planar maps. In Clay Mathematics Summer School 2010. Available at arXiv:1101.4856. MR2730798

Lindvall, T. (1972). Convergence of critical Galton-Watson branching processes. J. Appl. Probab. 9 , 445-450. MR0345227

Lindvall, T. (1974). Limit theorems for some functionals of certain Galton-Watson branching processes. Adv. in Appl. Probab. 6, 309-321. MR0345228

Malyshev, V., Yambartsev, A. and Zamyatin, A. (2001). Two-dimensional Lorentzian models. Mosc. Math. J. 1, 439-456. MR1877603

Napolitano, G. M. and Turova, T. (2016). The Ising model on the random planar causal triangulation: Bounds on the critical line and magnetization properties. J. Stat. Phys. 162, 739-760. MR3451503

Neveu, J. (1986). Arbres et processus de Galton-Watson. Ann. Inst. H. Poincaré Probab. Statist. 22, 199-207. MR0850756

Pakes, A. G. (1999). Revisiting conditional limit theorems for the mortal simple branching process. Bernoulli 5, 969-998. MR1735780

Sisko, V., Yambartsev, A. and Zohren, S. (2013). Growth of uniform infinite causal triangulations. J. of Stat. Phys. 150, 353-374. MR3022463

V. Sisko

Department of Mathematics, UFF

Rua Mario Santos Braga, s/n-Centro

Niterói-RJ, 24020-140

Brazil

E-mail: valentin.sisko@id.uff.br

\author{
A. Yambartsev \\ Institute of Mathematics and Statistics, USP \\ Rua do Matão, 1010_-Vila Universitaria \\ São Paulo—SP, 05508-090 \\ Brazil \\ E-mail: yambar@ime.usp.br
}

\author{
S. Zohren \\ Department of Materials \\ University of Oxford \\ Parks Road \\ Oxford OX1 3PH \\ United Kingdom \\ E-mail: stefan.zohren@materials.ox.ac.uk
}

